\title{
Editorial
}

\section{Scheduling with Time-Dependent Processing Times 2015}

\author{
Yunqiang Yin, ${ }^{1}$ T. C. E. Cheng, ${ }^{2}$ and Chin-Chia $W u^{3}$ \\ ${ }^{1}$ Faculty of Science, Kunming University of Science and Technology, Kunming 650093, China \\ ${ }^{2}$ Department of Logistics and Maritime Studies, The Hong Kong Polytechnic University, Hung Hom, Kowloon, Hong Kong \\ ${ }^{3}$ Department of Statistics, Feng Chia University, Taichung 40724, Taiwan
}

Correspondence should be addressed to Yunqiang Yin; yunqiangyin@gmail.com

Received 8 June 2015; Accepted 8 June 2015

Copyright (C) 2015 Yunqiang Yin et al. This is an open access article distributed under the Creative Commons Attribution License, which permits unrestricted use, distribution, and reproduction in any medium, provided the original work is properly cited.

Scheduling with time-dependent processing times is concerned with scheduling situations in which the job processing times vary with some situational parameters. Research on time-dependent scheduling problems has spawned a new area in the scheduling field. It has uncovered many new properties that are absent in classical scheduling theory and led to new methodological approaches to algorithmic design and NP-hardness proof. Due to its theoretical challenge and practical value, scheduling with time-dependent processing times has attracted a considerable amount of research attention in the literature. We publish in this issue a number of state-of-the-art studies on the topic that span the singlemachine, flow shop, and parallel settings.

For research involving learning and/or deteriorating jobs on a single machine, X. Zhang and Q. Xie consider single-machine scheduling with job ready times under the assumptions of group technology and position-dependent processing times. Their objective is to find the optimal group sequence and the optimal job sequence to minimize the makespan. P. Ji and L. Li consider single-machine group scheduling with general position-dependent learning effects and deteriorating jobs simultaneously. Under the assumption that the group setup time is a positive strictly decreasing continuous function of the amount of consumed resource, they show that the makespan minimization problem is polynomially solvable under the constraint that the total resource consumption does not exceed a given limit, and the total resource consumption minimization problem is also polynomially solvable under the constraint that the makespan does not exceed a given limit. J.-G. Kim et al. consider the lotorder assignment problem with the objective of minimizing the total tardiness of the orders with distinct due dates. They show that the proposed problem can be solved optimally by finding an optimal sequence for the single-machine total tardiness scheduling problem with nonnegative time-dependent processing times. Assuming that the actual processing times of all the jobs are subject to a sum-of-processing-timesbased aging effect, D. G. Kim and J. Y. Choi consider a twoagent single-machine scheduling problem that minimizes the total weighted tardiness of one agent under the restriction that the second agent is prohibited from having tardy jobs. S.-C. Liu et al. investigate a single-machine problem with sum-of-processing-times-based learning and ready times to minimize the makespan. To solve the problem, they build a branch-and-bound algorithm and a heuristic algorithm to find the optimal solution and near-optimal solution, respectively. T. Wang and $\mathrm{D}$. Xu consider a maximum lateness minimization scheduling problem on a single machine with a mandatory maintenance whose duration is workload dependent. They show that the problem is NP-hard and propose an approximation algorithm for it. $\mathrm{K}$. Li and $\mathrm{H}$. Tian investigate a new variant of the single-machine scheduling problem by considering sequence-dependent setup costs and demands of downstream production lines. They formulate it as a mixed integer linear programming model and propose an improved particle swarm optimization to solve it. Y. Qi et al. investigate a scheduling problem on a single machine with a maintenance, in which the starting time of the maintenance is given in advance but its duration depends on the load of the machine before the maintenance. They formulate it as an integer programming model, discuss the complexity, and propose an FPTAS and fast heuristic algorithms for the problem. 
Three papers study parallel-machine or multiple-machine scheduling with deteriorating jobs or learning effects or both. C. Miao and J. Zou consider parallel-machine scheduling in which the machines have availability constraints and the processing time of each job is a simple linear increasing function of its starting times. They discuss the complexities for the makespan, total completion time, and total weighted completion time minimization problems. W. Ma et al. consider parallel-machine scheduling with past-sequence-dependent delivery times and aging maintenance. They develop polynomial algorithms for three versions of the problem of minimizing the total absolute deviation of job completion times, the total load, and the total completion time. Y. Zhou and Q. Zhang study cooperative games based on multiplemachine scheduling with learning effects.

In the flow shop setting, G.-C. Lee et al. propose a hybrid method that combines the beam search and NEH method for two-stage hybrid flow shop scheduling with sequencedependent setup times and compare the performance with other existing local search methods. R.-H. Huang et al. consider a no-wait flow shop with multiprocessors with a time window constraint and propose an improved ant colony optimization to solve the problem.

Six potential topics include the papers discussing a compensated numerical method for solving stochastic differential equations with variable delays and random jump magnitudes by Y. Du and C. Mei, investigating individual traffic police on duty behaviour analysis method with time series scheduling by $\mathrm{H}$. Peng et al., addressing agent behaviour-based simulation on the mass collaborative product development process by X. Zhang et al., studying a novel model for lattice-based authorized searchable encryption with special keywords by F. Zeng and $\mathrm{C}$. Xu, investigating online scheduling with delivery times on a bounded parallel batch machine with limited restart by $\mathrm{H}$. Liu et al., and addressing a spatial resource constrained project scheduling problem by S. Hu et al.

We hope these papers help readers to further explore research on scheduling with time-dependent processing times and related topics.

Yunqiang Yin

T. C. E. Cheng

Chin-Chia Wu 


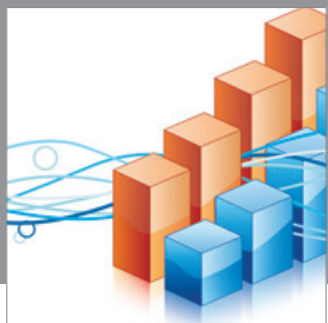

Advances in

Operations Research

mansans

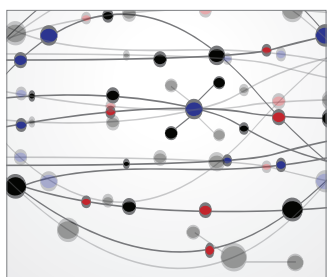

The Scientific World Journal
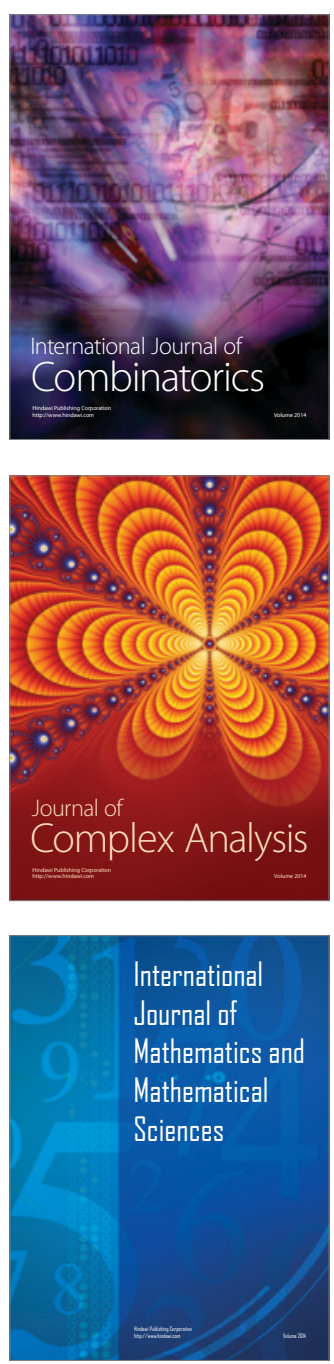
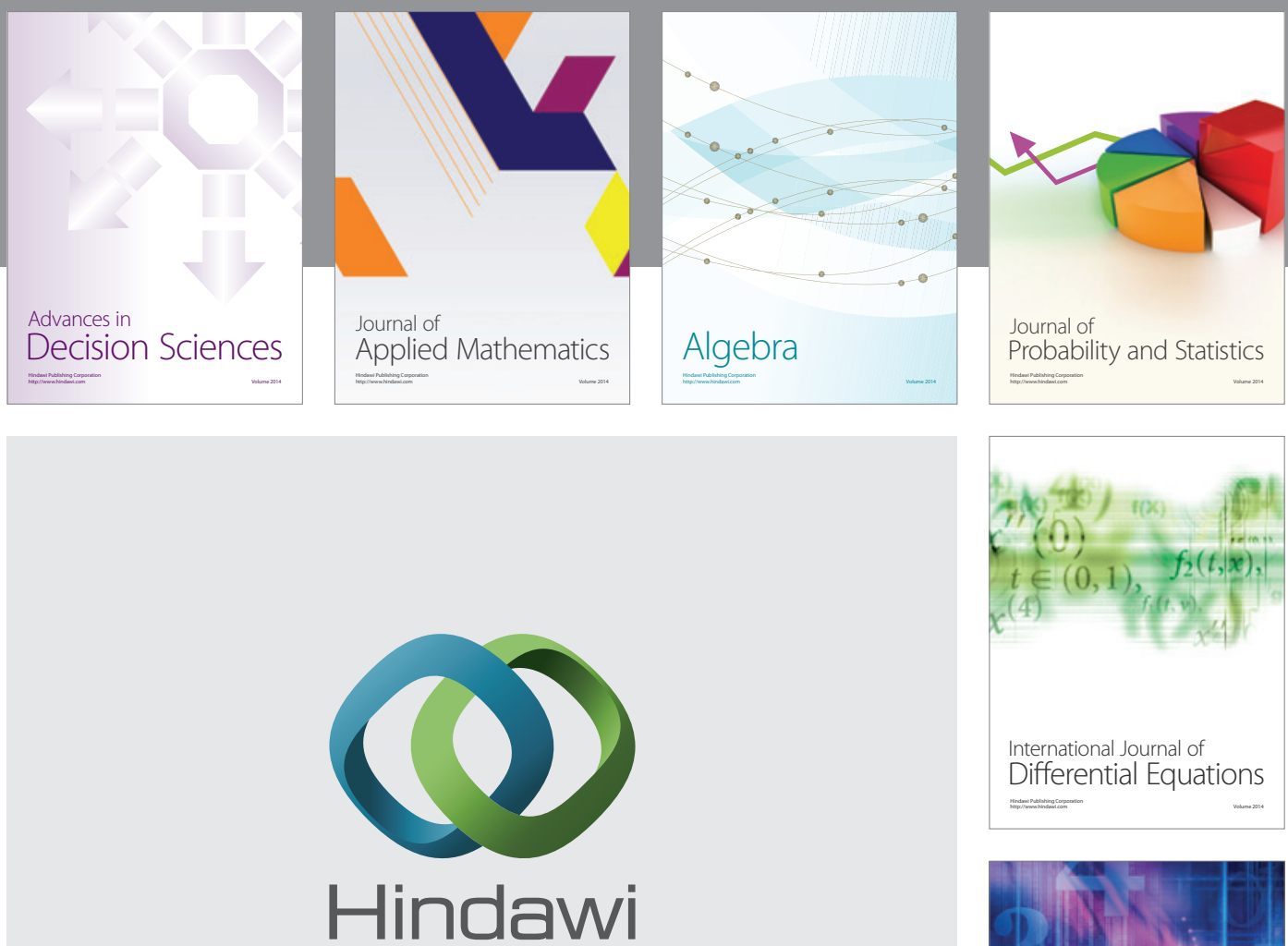

Submit your manuscripts at http://www.hindawi.com
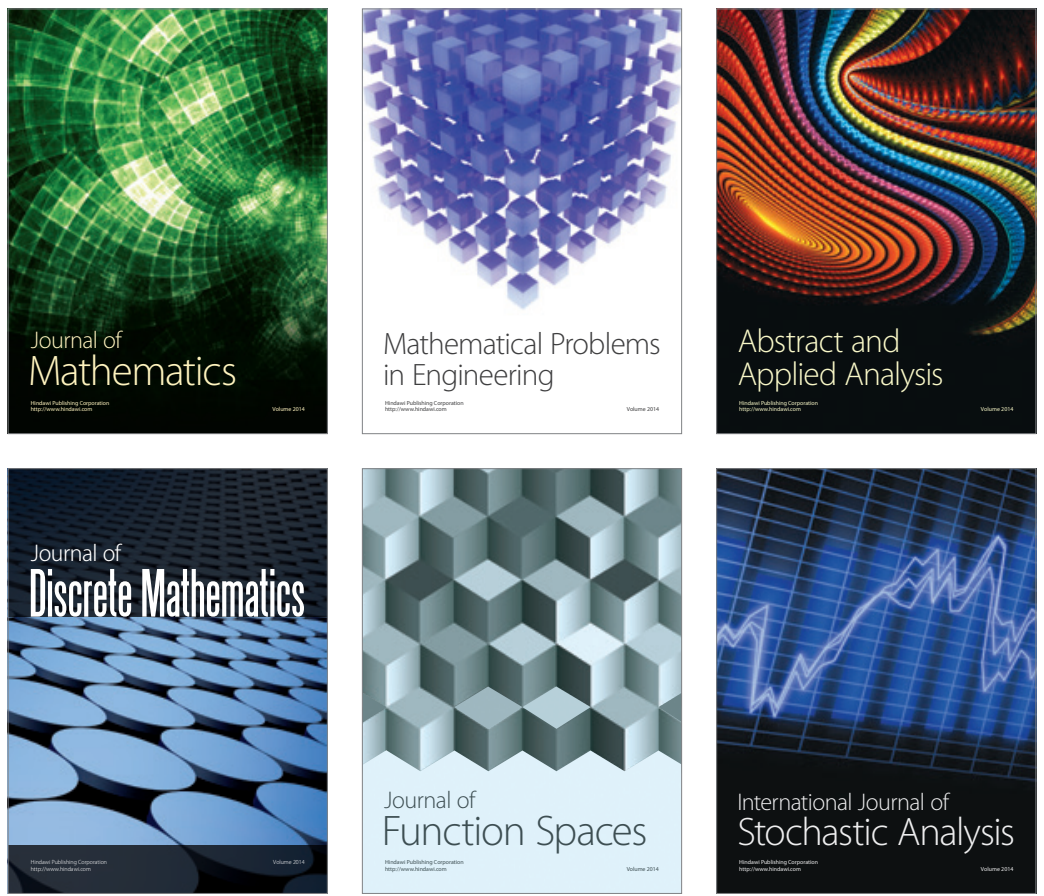

Journal of

Function Spaces

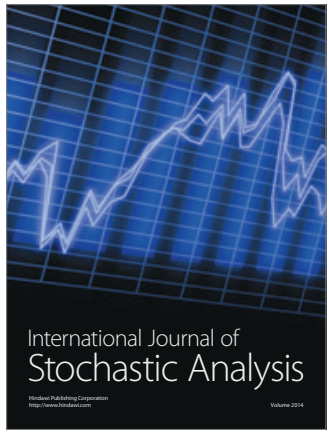

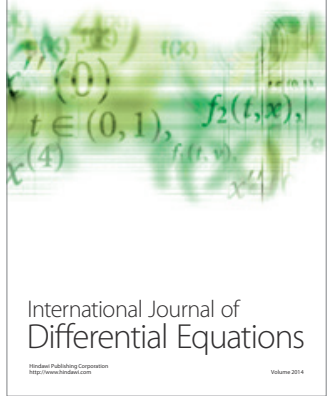
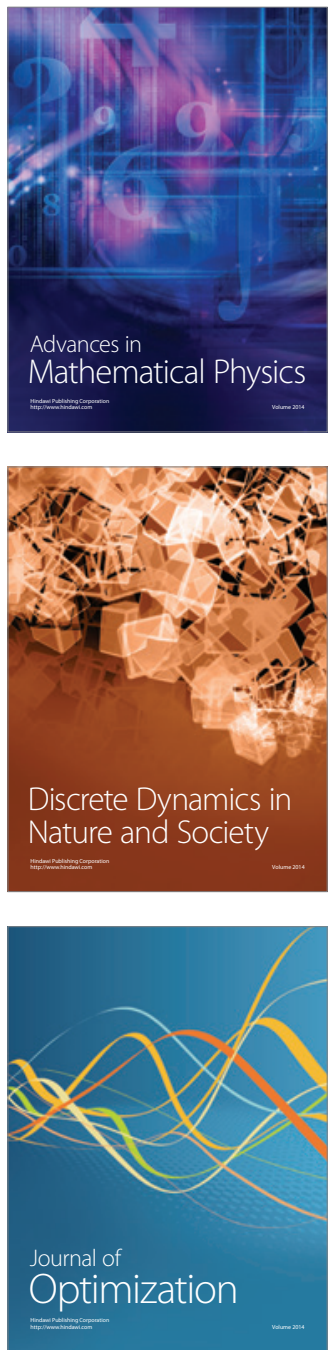